\title{
Predictores eCOlógICOS DE VIOLENCIA EN JÓVENES: COMUNIDAD, FAMILIA, PARES Y ESTADO EMOCIONAL
}

\author{
Elisa Rodríguez Maldonado \\ Universidad de Puerto Rico \\ Puerto Rico
}

\begin{abstract}
RESUMEN
Basándose en los supuestos de Bronfenbrenner (1994), el estudio exploró si un arreglo estructural de factores de distintos niveles contextuales se ajusta a lo esperado teóricamente, para explicar la conducta violenta en una muestra de 431 jóvenes de la Región Educativa de Cidra. Se especificó un modelo de rutas en el que se estimaron los efectos de las variables, "percepción de violencia en la comunidad", "ausencia de violencia intrafamiliar" e "influencia negativa de los pares". Se estudió además el efecto mediado por "inestabilidad emocional". En los hallazgos, la varianza explicada para la conducta violenta fue de $\mathrm{R}^{2}=0.54$, y para la inestabilidad emocional fue de $\mathrm{R}^{2}=0.28$, lo que muestra la alta influencia de estas variables en su conducta violenta. Se discute la dirección y la magnitud del efecto de cada factor y la necesidad de incluirlos en el diseño de estrategias para la prevención de violencia en jóvenes.
\end{abstract}

\section{Palabras Clave:}

violencia intrafamiliar, jóvenes, inestabilidad emocional, Bronfenbrenner, modelo ecológico.

\section{Ecological Predictors of Youth Violence: Community, Family, PeERs, and EMOTIONAL STATE}

\begin{abstract}
Based on the ecological assumptions of Bronfenbrenner, the study explored whether a structural arrangement of factors at different contextual levels are in line with what was theoretically expected, to explain violent behavior in a sample of 431 students from the Cidra Educational Region. A path analysis model was specified, in which effects on young people's violent behavior were estimated for three variables, "perception of violence in the community", "absence of intrafamily violence" and "negative influence of peers ". The mediator effect of the "emotional instability" was also studied. The results indicated that all the factors of the model contributed significantly, explaining $54 \%$ of the variance of violent behavior, and the $28 \%$ of emotional instability. The direction and magnitude of each factor, and the need to include these factors in the design of strategies for youth violence prevention and other implications for practice is discussed.
\end{abstract}

\section{Keywords:}

family violence, youth, emotional stability, Bronfenbrenner, ecological model

Bitácora del Artículo:

| Recibido: 1 de Febrero de 2018 | Aceptado: 1 Abril de 2018 | Publicado en línea: Enero-Junio de 2018 | 


\title{
PREDICTORES ECOLÓGICOS DE VIOLENCIA EN JÓVENES: COMUNIDAD, FAMILIA, PARES Y ESTADO EMOCIONAL
}

\author{
Elisa Rodríguez Maldonado
}

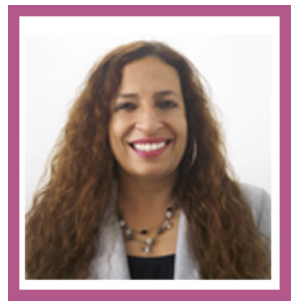

Elisa Rodríguez Maldonado

Universidad de Puerto Rico

Correo: elisa.rodriguez3@upr.edu

Doctora en Filosofía de las Artes, con concentración en Psicología, de la Universidad de Puerto Rico. Actualmente se desempeña como catedrática auxiliar en la Universidad de Puerto Rico en Cayey, Facultad de Psicología, Departamento de Ciencias Sociales.

Ver más...

\section{CONTRIBUCIÓN DE lOS AUtORES}

La Dra. Elisa Rodríguez-Maldonado es responsable por el diseño y ejecución de la investigación, así como del análisis estadístico y discusión de los resultados.

\section{AgRADECIMIENTOS}

Esta investigación fue posible gracias al trabajo voluntario de estudiantes de múltiples semestres del curso INTD 4116 de la Universidad de Puerto Rico en Cayey, y también gracias a la colaboración de los miembros de las comunidades y escuelas participantes. En particular, es mi deber expresar mi agradecimiento al personal de la desaparecida COPREVI (Comisión para la Prevención de la Violencia) por tan amablemente permitirnos utilizar sus instalaciones y equipos para reuniones y el procesamiento de datos. Además, reconozco la colaboración de mis principales asistentes de investigación Aidymar Rivera Rodríguez, estudiante doctoral en la Ponce School of Medicine, e Inés Herrero Guzmán, estudiante doctoral en la Boston Graduate School of Psychoanalysis. Finalmente, agradezco el respaldo de mi compañero, y el apoyo de mis hijos, Andrés, y Hani, ustedes son la alegría de mis días y mi mayor inspiración para continuar investigando las dinámicas de la violencia en Puerto Rico, en el camino de trabajar hacia una sociedad puertorriqueña más justa y solidaria.

\section{Datos de Filiación de LA Autora}

Universidad de Puerto Rico en Cayey, Facultad de Psicología, Departamento de Ciencias Sociales.

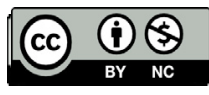

Copyright: (c) 2018 Rodríguez-Maldonado, E.

Este es un artículo de acceso abierto distribuido bajo los términos de la licencia Creative Commons Reconocimiento-NoComercial 4.0 Internacional, por lo que su contenido gráfico y escrito se puede compartir, copiar y redistribuir total o parcialmente sin necesidad de permiso expreso de su autor con la única condición de que no se puede usar con fines directamente comerciales y los términos legales de cualquier trabajo derivado deben ser los mismos que se expresan en la presente declaración. La única condición es que se cite la fuente con referencia a la Revista Digital Internacional de Psicología y Ciencia Social y a su autor. 


\section{TABLA DE CONTENIDO}

INTRODUCCIÓN

ESTUPIAR CONDUCTA VIOLENTA DESDE LOS MODELOS SISTÉMICOS ECOLó-̄i
GIOS

ACEPCIONES DE LA CONDUCTA VIOLENTA EN JÓVENES

COMUNIDAD, FAMILIA, PARES, ESTADO EMOCIONAL Y LA CONDUCTA VIO-63

MÉTODO

Participantes, 65

Variables demográficas, 66

Materiales, 66

Procedimiento, 67

Mediciones, 67

ANÁLISIS ESTADÍSTICOS

RESULTADOS

Discusión 


\section{INTRODUCCIÓN}

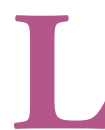

a conducta violenta en jóvenes es un fenómeno que presenta múltiples complejidades y variantes. En Puerto Rico se ha intensificado la violencia entre jóvenes en los últimos 10 años, viéndose éstos involucrados en muertes, atentados contra la vida, escalamientos y otros actos violentos (Instituto de Estadísticas de Puerto Rico, 2015). Muchas veces estos actos han estado relacionados con las dinámicas que origina el mundo del narcotráfico, la crisis de valores prosociales, la desigualdad social y otras causas que se reproducen en las diversas esferas en que se desarrolla el joven, como la familia, los grupos de pares y la comunidad. Son precisamente estos nichos ecológicos, familia, grupos de pares y comunidad, áreas esenciales a las cuales prestar atención al momento de diseñar intervenciones para prevenir violencia en estas poblaciones.

En el presente estudio se exploró la influencia de estos factores de niveles sistémicos distintos (la percepción de violencia en la comunidad, la ausencia de violencia en la familia, la influencia negativa de los pares y la inestabilidad emocional) en la conducta violenta en jóvenes. Deseábamos evaluar si la presencia o ausencia de violencia en estos niveles sistémicos ejerce una influencia importante en el desarrollo de conductas violentas en la adolescencia, y conocer cuál es la magnitud de dichas influencias. Se pretendió analizar los efectos directos de estos factores en la conducta violenta y su influencia mediada por el estado emocional del joven. Con esta finalidad, se midió, mediante un cuestionario, el comportamiento de estas condiciones contribuyentes a la conducta violenta en jóvenes en el distrito escolar de la región de Cidra, en Puerto Rico. El objetivo consistió en probar si un arreglo estructural de influencias de factores se ajusta a lo esperado teóricamente, según lo reportado en la literatura respecto al tema. Se espera obtener evidencia de qué factores en la ecología del individuo influyen en su conducta violenta (Hong, Kral, Espelage y Allen-Meares, 2012; Swick y Williams, 2006; Bronfenbrenner, 1994).

\section{ESTUDIAR CONDUCTA VIOLENTA DESDE LOS MO-}

\section{DELOS SISTÉMICOS ECOLÓGICOS}

Son numerosos los acercamientos teóricos que se han elaborado acerca de las nociones de sistemas y sus leyes de funcionamiento. Ya desde la década de 1940, la teoría general de los sistemas del biólogo y filósofo austrohúngaro Von Bertalanffy (1968) impulsó la tendencia de la investigación científica a trascender las explicaciones mecanicistas de finales del siglo XIX y principios del XX respecto a los fenómenos humanos. En esta tradición fundadora de los estudios de sistemas vale la pena mencionar al sociólogo alemán Luhmann, a los biólogos y filósofos chilenos Maturana y Varela, y al psicólogo rusoestadounidense Bronfenbrenner. Estos destacados pensadores de base argumentaron en su época la necesidad de ampliar la mirada y atender las interacciones dialécticas de procesos, comunicación y significado, de lo sistémico.

El modelo ecológico propuesto por Urie Bronfenbrenner (1994) es quizás uno de los que mejor expone estas interacciones entre niveles micro, intermedio y macro de influencias y transacciones entre los factores de la conducta y el desarrollo humano. El modelo ecológico general de Bronfenbrenner asume dos proposiciones fundamentales (Bronfenbrenner, 1994):

1. El desarrollo humano se lleva a cabo a través de procesos de interacciones recíprocas progresivamente complejas entre un organismo humano activo en evolución biopsicológica y las personas, objetos y símbolos en su ambiente inmediato, por un periodo extendido, de forma regular (denominados procesos proximales).

2. La forma, poder, contenido y dirección de estos procesos proximales que efectúan el desarrollo varían sistemáticamente como una función conjunta de las características de la persona en desarrollo, del ambiente (tanto inmediato como remoto), en los cuales se está llevando a cabo el proceso, y la naturaleza de los resultados de desarrollo en consideración.

Con estos dos postulados principales resume Bronfenbrenner toda una serie de trabajos que fundamentan su teoría ecológica del desarrollo y que suministra la guía conceptual que enmarca esta investigación. El diseño de investigación que permite el estudio simultáneo de estas transacciones sistémicas se conoce como modelo proceso-persona-contexto, donde el poder de los procesos proximales varía de manera sistemática en función del contexto ambiental (violencia comunitaria, familiar, en los pares) y las características de la persona. También los procesos proximales tienen el efecto general de servir como reductores o amortiguadores contra las características del ambiente de niveles superiores, $\mathrm{o}$, de forma contraria, amplificadores de los efectos en el resultado de la conducta de la persona.

Basándose en la teoría de campo de Lewin, Bronfenbrenner plantea su modelo explicativo del desarrollo 
en que diferentes áreas de influencia quedan contenidas unas dentro de otras (Bronfenbrenner, 1994). Al nivel de influencias más inmediato de la persona en desarrollo le denomina microsistema, donde incluye las interacciones entre el individuo y su entorno inmediato. Éstas contienen las relaciones de cara a cara entre el individuo y su familia, escuela, amigos y otros. En un segundo nivel de interacciones ubica al mesosistema, el cual consiste en las interacciones entre dos o más microsistemas, como sería la relación que puede existir entre la familia y las influencias de los pares. El exosistema es el tercer nivel del modelo y comprende los nexos y procesos que ocurren entre dos o más escenarios, con la característica de que en uno de ellos no está presente la persona en desarrollo. Ejemplo de un exosistema es la relación entre la violencia familiar y los contextos vecinales o comunitarios en que se encuentre inmerso un adolescente. Un cuarto nivel, denominado macrosistema, evidencia el patrón general de microsistemas, mesosistemas y exosistemas que distinguen a la cultura del contexto estudiado, como la violencia en la sociedad. Por último, Bronfenbrenner extiende los niveles de análisis al cronosistema, donde distingue la importancia de incorporar los cambios y consistencias en el tiempo que presenta no sólo la persona, sino el contexto histórico y los eventos sociales en el que ésta se desarrolla.

Las dinámicas de influencia de los niveles ecológicos en las personas tienen lugar en dos campos: el de la percepción y el de la acción. Para Bronfenbrenner, en el campo de la percepción se observa en qué medida el punto de vista que tiene de la sociedad el individuo incluye otros entornos en los que ha participado activamente, las relaciones entre estos escenarios, la naturaleza y la influencia de los contextos externos con que no ha tenido contacto inmediato, y los patrones de organización social, creencias y estilos de vida específicos de la cultura (Bronfenbrenner, 2002). El campo de la acción se refiere a la capacidad, o incapacidad, de la persona para utilizar estrategias efectivas, para tener retroalimentación de los sistemas de niveles lejanos, para que estos sistemas continúen funcionando, y para reorganizar los sistemas existentes, o crear nuevos, de órdenes comparables o superiores, que se adecúen a sus deseos y necesidades (Bronfenbrenner, 2002). De esta manera, Bronfenbrenner explica a nivel ontogénico la presencia de elementos cuyo origen pueden ser más distales al individuo.

En resumen, la teoría ecológica social postula varios puntos principales (Voith, Gromoske y Holmes, 2014; Boxer, Huesmann, Dubow, Landau, Gvirsman, Shikaki y Ginges, 2013; Bronfenbrenner, 1977). En pri- mer lugar, los factores en los niveles más distales al individuo tienen una influencia más débil en el desarrollo. En segundo lugar, los factores más proximales al individuo tienen una mayor influencia. En tercer lugar, no hay un solo factor que por sí mismo determine el desarrollo (Bronfenbrenner y Crouter, 1983). Los jóvenes que han experimentado violencia familiar la han experimentado en el microsistema porque la violencia fue dirigida a ellos. Como se ha mencionado, el mesosistema incluye interacciones de dos o más microsistemas y se considera una posición distal de segundo orden en relación con el microsistema. Un joven que ha sido testigo de violencia familiar - y esta condición afecta a su vez la influencia que tienen sus pares - habría estado expuesto a la violencia en este nivel (Frías y Gaxiola, 2008). Los exosistemas se consideran parte de las estructuras sociales de orden superior, y se ubica a dos posiciones de distancia del individuo. Por ejemplo, vivir en una comunidad insegura con una elevada tasa de delincuencia podría considerarse una forma de exposición a la violencia de este nivel, aunque los efectos en el joven pueden puncionar por medio de factores en otros niveles. A estos objetivos, en lugar de medir la violencia acumulativa por el número de tipos de violencia que se vive, la violencia acumulativa se puede medir por el número de ámbitos ecológicos en los que una persona puede estar expuesta a la violencia (Voith, Gromoske y Holmes, 2014).

El estudio incluyó factores de nivel microsistémico, mesosistémico y exosistémico, planteando que las características del entorno afectan positiva o negativamente las manifestaciones de violencia en los jóvenes, y pretendemos explorar la magnitud de estas influencias directas. En los niveles microsistémicos indagamos acerca del efecto que puede tener un ambiente familiar libre de violencia en la conducta de los jóvenes, así como respecto a las influencias que pueden aportar los grupos de pares que presentan conductas violentas. Entendemos que la contribución de la ausencia de violencia en la familia y la influencia negativa que pueda tener el grupo de pares en la conducta violenta de los jóvenes estará mediada por características a nivel ontogénico, como la inestabilidad emocional de la persona. En el nivel mesosistémico proyectamos una asociación entre los factores de microsistemas: ausencia de violencia en la familia e influencias negativas de los pares. Además, proponemos que la violencia percibida en la comunidad, de nivel exosistémico, tiene un efecto en la conducta violenta, tanto directo como mediado por la inestabilidad emocional del individuo. La percepción de violencia en la comunidad también estará asociada a factores de microsistemas: ausencia de violencia en la familia e influencias negativas de los pares. 


\section{ACEPCIONES DE LA CONDUCTA VIOLENTA EN JÓVENES}

En ocasiones se utiliza la frase "conducta violenta" como sinónimo de otros conceptos. Veamos algunos ejemplos. Aunque guardan algunas congruencias, los términos "trastorno de conducta" o "trastorno de personalidad antisocial" hacen referencia a las implicaciones clínicas-psicopatológicas que pueden estar presentes o no en las conductas violentas. Dicho de otra manera, para que haya un diagnóstico clínico tienen que cumplirse criterios de deterioro significativo de las actividades sociales, académicas o laborales del individuo.

De la misma manera, la noción de "conducta delictiva" se apoya en el marco jurídico, donde infringir y violentar las leyes y normas sociales es el criterio. Por su parte, la acepción sociológica de "conducta desviada" implica a "las conductas, ideas o atributos que ofenden (disgustan o perturban) a los miembros de una sociedad (aunque no necesariamente a todos)" (De la Peña-Fernández, 2010). En este sentido, la desviación no es una cualidad intrínsecamente ligada a ningún tipo de acto, sino que una determinada conducta podrá categorizarse como "desviada" sólo con referencia a un contexto normativo, social y situacional definido (De la Peña-Fernández, 2010). Nuestra definición de conducta violenta en jóvenes propone entonces abarcar conductas que producen daño físico o emocional a personas, incluyendo a sí mismo, o daño a propiedades, independientemente de que haya repercutido o no en consecuencias legales o médicas.

En este trabajo hemos denominado "conducta violenta en jóvenes" a cualquier forma de conducta física o verbal dirigida a dañar o agraviar, independientemente de que se presente con animosidad o como vehículo para lograr un objetivo, incluyendo, pero no limitándose a, lo que en estudios relacionados se ha englobado bajo el concepto de conducta antisocial (Burnette, Oshri, Lax, Richards y Ragbeer, 2012; Slattery y Meyers, 2014). De este modo nos referimos además como conducta violenta o antisocial a cualquier tipo de conducta que conlleve el infringir las reglas o normas sociales y que represente una acción contra los demás, independientemente de su gravedad o de las consecuencias que a nivel jurídico puedan acarrear (De la Peña-Fernández, 2010). En este caso preferimos utilizar el término "conducta violenta" para hacer la distinción de lo que constituyen definiciones que guardan cierta similitud, como "desorden de conducta", "trastorno de personalidad antisocial", "conducta delictiva" y "conducta desviada", pero que limitan el campo de estudio a la psiquiatría, la criminología o la sociología.

\section{COMUNIDAd, FAMILIA, PARES, ESTADO EMOCIO-} NAL Y LA CONDUCTA VIOLENTA EN JÓVENES

En la literatura psicológica mencionada encontramos la conducta violenta en jóvenes analizada tanto a partir de un factor específico único como en su relación con algunos escenarios de microsistemas. Estudios previos Salzinger, Ng-Mak, Feldman, Kam y Rosario, 2006). El nivel de atención, los problemas de aprendizaje, el comportamiento antisocial, la desesperanza, presenciar violencia y ser víctima de violencia son algunos de los factores estudiados que han sido asociados con niveles altos de agresión y violencia en jóvenes (Cedeño, Elia, Kelly y Chu, 2010; Stoddard, 2012). Por otro lado, factores a nivel individual, como habilidades sociales, rendimiento escolar, involucramiento con la escuela y un sentido de esperanza y propósito, han sido considerados promotores de conductas no violentas (Borowsky, Widome \& Resnick, 2008; Cedeño et al., 2010; Durant, Cadenhead, Pendergrast, Slavens \& Linder, 1994; Farrell y Sullivan, 2010).

Diferentes investigaciones han explorado los efectos de factores - como la violencia en la comunidad, la violencia en la familia, la influencia de los pares y el estado emocional-que se estima influyen en el desarrollo de la conducta violenta o antisocial en la juventud. Slattery y Meyers (2014) identifican tres factores que afectan el desarrollo de conducta antisocial en jóvenes. Estos investigadores, entre otros, plantean la existencia de una asociación entre el bajo nivel de supervisión de los padres (Burnette, Oshri, Lax, Richards y Ragbeer, 2012; Graber, Nichols y Lynne, 2006), la afiliación del adolescente con compañeros problemáticos (Keenan, Wroblewski, Hipwell, Loeber y Stouthamer-Loeber, 2010; Salzinger, Ng-Mak \& Feldman, 2006) y la exposición de los adolescentes a la violencia dentro de su comunidad (Farrell y Sullivan, 2004; Gorman-Smith, Henry y Tolan, 2004) con el desarrollo de conductas antisociales violentas. El involucramiento con pares violentos y la exposición a violencia comunitaria probaron ser factores importantes de la conducta violenta en jóvenes en este estudio. No obstante, en sus resultados finales, el monitoreo de los padres y el ambiente familiar no se reflejó como un predictor importante de conducta antisocial distinto a sus expectativas. Estos investigadores explican que en un tipo de conducta violenta visible, directa y confrontacional, el monitoreo de los padres no se relacionó con menor grado de violencia manifestada, aunque sí mostró algún grado de influencia en un tipo de violencia más sutil e indirecta. Los autores atribuyen la poca influencia de la supervisión de los padres y el buen ambiente familiar en las manifestaciones de con- 
ducta antisocial de sus hijos a un componente reactivo e impulsivo de los actos agresivos, que alude a la falta de control emocional, aún en presencia de elementos moduladores, como el monitoreo de los padres.

La violencia intrafamiliar es un factor que se presenta en la literatura relacionado con manifestaciones de conducta violenta en jóvenes. Tanto las experiencias de violencia directa recibidas en el núcleo familiar (haber sido objeto de maltrato físico o emocional), como las de violencia indirecta (haber presenciado episodios de violencia entre los padres) parecen aportar al desarrollo de inestabilidad emocional problemas psicológicos, dificultades escolares y conducta violenta en la adolescencia. Para Frías y Gaxiola "la violencia familiar produce problemas de ajuste conductual, social y emocional que se traducen en conducta antisocial en general, depresión y ansiedad, y problemas en la escuela" (Frías y Gaxiola, 2008: 238). Tres décadas de investigación sobre maltrato en niños: implicaciones para los años escolares [traducción de la autora] fue una extensa revisión de estudios científicos publicada en 2001 por Veltman y Browne. En ella se demuestra que en $75 \%$ de las investigaciones entre 1966 y 1999, el maltrato infantil en la familia se asociaba con el retraso en el desarroIlo cognitivo o intelectual, con el rezago en el lenguaje y con un pobre rendimiento académico (Veltman y Browne, 2001). Las experiencias de violencia intrafamiliar en la niñez incrementan la probabilidad de problemas de conducta en la edad escolar, acompañados de comportamiento opuesto a las reglas y pobres destrezas de autocontrol, características asociadas de manera consistente con conductas violentas (Resnicow, Ross-Gaddy y Vaughan, 1995). A su vez, los bajos índices de rendimiento académico y los problemas escolares asociados con proceder de un hogar violento se relacionan con la conducta antisocial, tanto en los años de adolescencia como en la etapa adulta (Frías y Gaxiola, 2008).

La exposición a la violencia en la comunidad también se ha asociado de manera recurrente con comportamientos agresivos, ambos situacionales y personales. En la adaptación a las comunidades violentas o barrios peligrosos, los jóvenes pueden usar la agresión para poner orden en ambientes impredecibles y ver la agresión como método eficaz para producir resultados deseables (McMahon et al., 2013; Latzman y Swisher, 2005).

Margolin, Vickerman, Oliver y Gordis (2010) analizaron los efectos de la exposición a diversos tipos de violencia (agresión física a los jóvenes, violencia conyugal y violencia en la comunidad) en diferentes dominios interpersonales de los jóvenes. Encontraron que mientras que algunos síntomas adversos en jóvenes estaban relacionados con varios dominios de la exposición a la violencia, otros fueron más claramente relacionados con un dominio específico de la violencia. El desempeño en la escuela se vio afectado por la exposición a cualquiera de los dominios de violencia. Las conductas delictivas se relacionaron de manera significativa a la violencia intrafamiliar, sobre todo a la agresión de los padres dirigida a los jóvenes y a la agresión física conyugal. La ansiedad y la inestabilidad emocional se relacionaron sólo con la agresión conyugal física, derivada de las preocupaciones de los jóvenes por la falta de control en el conflicto, y por sus preocupaciones por su propia seguridad y por el bienestar de la familia. La violencia en el hogar entre los padres, donde los jóvenes fueron testigos, predijo conductas de internalización en ellos (inestabilidad emocional), mientras que la disciplina física predijo conductas de externalización (conducta violenta). La violencia comunitaria se relacionó a niveles altos de sobreexcitación en los jóvenes y contribuyó a los efectos de los otros tipos de violencia, manisfestada en síntomas de depresión en los adolescentes, inestabilidad emocional y dificultades escolares (Margolin, Vickerman, Oliver y Gordis, 2010).

En 2014 los investigadores Voith, Gromoske y Holmes revisaron la investigación de Margolin et al. (2010) y propusieron un análisis más detallado de los efectos acumulados de la exposición a diferentes tipos de violencia, argumentando que los niños y jóvenes expuestos a múltiples formas de violencia (como victimización directa y exposición a violencia familiar) han demostrado sintomatología más compleja, en comparación con los expuestos a una sola forma de violencia (Voith, Gromoske y Holmes, 2014). La polivictimización o exposición a violencia acumulada se define como la exposición a múltiples formas de violencia (Finkelhor, Ormrod y Turner, 2007). Voith, Gromoske y Holmes (2014) discutieron la importancia de ampliar la conceptualización teórica de la violencia y estudiaron los efectos de la violencia acumulada y la exposición a ella por zona ecológica, es decir, los efectos diferenciales de la victimización directa, la exposición a la violencia familiar y a la vida en una comunidad violenta. Estos autores indican a la necesidad de estudios adicionales acerca de los efectos de la violencia acumulada por medio de distintos niveles de influencia en la conducta.

Para estos fines explicativos, la investigación científica apoya perspectivas sistémicas y ecológicas, porque se sugiere que el comportamiento violento y/o prosocial están asociados tanto a factores individuales como a factores contextuales y sus transacciones (Cummings, Goeke-Morey, Merrilees, Taylor y Shirlow, 2014; Edwards y Bromfield, 2009; Stoddard, Zimmerman y Bauermeister, 2012; Swick y Williams, 2006). La evidencia sugiere que 
un vecindario con factores protectores contribuye al comportamiento prosocial (que beneficia a otras personas, o a la sociedad en general) (Edwards y Bromfield, 2009), pero es necesaria más investigación con diversas poblaciones para construir una mejor comprensión de los contribuyentes importantes al desarrollo de conducta violenta a través del tiempo. Algunos investigadores del tema (McMahon et al., 2013) explican que al presente no hay un consenso al examinar la relación entre exposición a la violencia y cómo ésta conduce al comportamiento antisocial o prosocial. Indican que existen estudios (Van der Merwe y Dawes, 2000, citado en McMahon et al., 2013) en los que se encontró que a pesar de los altos niveles de exposición a la violencia comunitaria, un mayor comportamiento agresivo y un déficit en la autorregulación, los jóvenes siguieron mostrando niveles relativamente altos de resultados prosociales. Por otra parte, en la adolescencia temprana suceden desarrollos del razonamiento cognitivo y moral de orden superior, que se esperaría estuvieran asociados con comportamientos prosociales (Stoddard et al., 2013). Mientras, otros autores del tema han encontrado una relación inversa entre la exposición a la violencia en la comunidad y el comportamiento prosocial (McMahon et al., 2013).

Quizás esta inconsistencia en resultados se deba a variables moduladoras que se han quedado fuera de las propuestas teóricas y que producen efectos importantes mediando en la relación entre exposición a violencia en la comunidad y las manifestaciones de conductas violentas en los jóvenes (Posada y Parales, 2012). Esta diversidad de conclusiones respecto a cómo afecta la violencia social y comunitaria indica la necesidad de investigación adicional que examine la relación entre los múltiples factores ecológicos y cómo se potencian, o no, por medio de los dominios de exposición e influencia (McMahon et al., 2013; Stoddard et al., 2012).

Como se ha indicado, el presente estudio tiene como objetivo examinar las interacciones e influencias de procesos individuales, familiares y sociales en la conducta violenta de adolescentes escolares de varias comunidades de Puerto Rico. Refleja una perspectiva orientada hacia los procesos socioecológicos, de acuerdo con los principios de la psicología del desarroIlo, incluyendo atención a los procesos, así como a los resultados, y diferenciando las influencias contextuales, tanto positivas como negativas. Como factor individual ontogénico analizamos la inestabilidad emocional de los jóvenes. En los factores de microsistemas verificamos la ausencia de violencia intrafamiliar y la influencia de los pares que manifiestan conducta violenta. A nivel de mesosistemas indagamos acerca de la relación entre es- tos escenarios microsistémicos. El nivel de exosistemas está representado por la percepción de la violencia comunitaria y su influencia en los sistemas más cercanos a los adolescentes. Estos enunciados de la perspectiva de desarrollo socioecológico se traducen en este trabajo en el modelo de la figura 1.

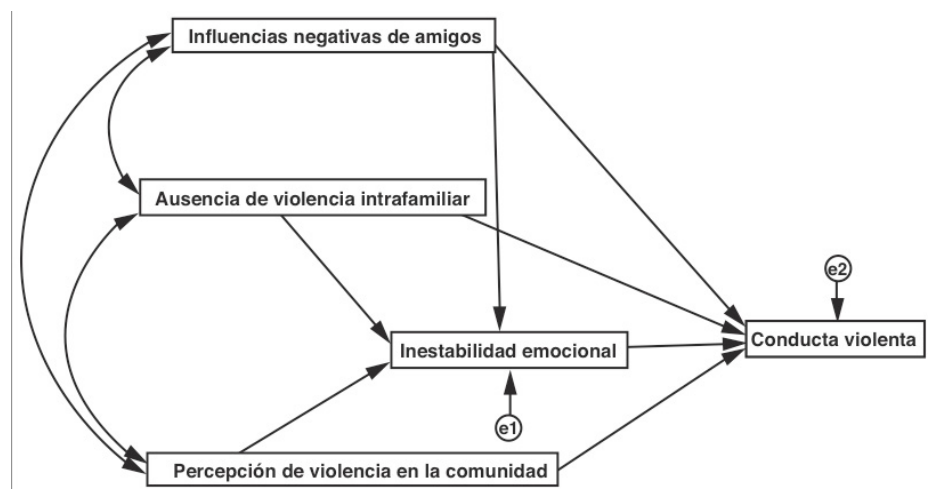

Figura 1.

Modelo teórico esbozando la ecología social de la conducta violenta para los factores incluidos en el estudio.

En la figura 1 se muestran las influencias e interacciones teorizadas entre variables exógenas y endógenas en dirección ontogénica, de acuerdo con el modelo ecológico. Como se ha establecido, es esperado en el modelo ecológico que la forma, poder, contenido y dirección de estos procesos transaccionales varíen sistemáticamente en función del tránsito dialéctico entre las características del entorno y de los actores en juego.

\section{Método}

\section{Participantes}

La muestra consistió de 433 estudiantes de escuela superior entre 14 a 18 años de edad, con una media de edad de 15.6. Participaron 263 mujeres y 170 hombres, de los grados décimo y undécimo (primer y segundo años de secundaria), de cuatro escuelas de la Región Educativa de Cidra, que comprende los pueblos de Cayey y Cidra, en Puerto Rico. Se seleccionó esta región del país por ser una zona donde en los últimos años se ha intensificado la violencia entre jóvenes, quedando en sexta posición en 2014 y en decimoquinta posición de 2015, de la totalidad de 78 municipios de la isla (IEPR, 2015), y por ser donde se encuentra localizado el centro universitario de donde surge esta investigación: la Universidad de Puerto Rico en Cayey. El acceso a las escuelas requirió la obtención de un permiso especial del Departamento de Educación de Puerto Rico. Se si- 
guieron todas las regulaciones éticas y de protección de los sujetos humanos en la investigación, incluyendo informar a los jóvenes que su participación era completamente voluntaria, que podían retirarse de la investigación en cualquier momento sin ninguna penalidad, $y$ que se protegería totalmente su confidencialidad. El estudio fue monitoreado y autorizado por la Junta Revisora Institucional de la Universidad de Puerto Rico en Cayey, comité que funciona como garante de la observación de las consideraciones éticas en nuestro recinto. Fue una muestra incidental por disponibilidad, no representativa, que, reconociendo sus limitaciones metodológicas, resulta apropiada para este tipo de estudio (Sampieri, Fernández, y Baptista, 2010). Los datos fueron recopilados durante el año escolar 2015-2016. Dos casos fueron eliminados por considerarse perdidos en relación con sus datos (los participantes no ofrecieron respuestas en más de $70 \%$ del cuestionario), por lo que la muestra final consistió de 431 participantes.

\section{Variables demográficas}

El $68 \%$ de la muestra son estudiantes que se encontraban en el nivel de pobreza, según las tablas de referencia de la Oficina del Censo de Estados Unidos, siendo el umbral de pobreza 23,624 dólares anuales para una familia de dos adultos y dos hijos(as) dependientes menores de 18 años (U. S. Census Bureau, 2012). El 27\% de la muestra provino de familias con ingresos menores a 5,000 dólares anuales, 47\% ingresaba 10,000 dólares o menos, y $68 \%$ recibe 20,000 dólares o menos al año (figura 2). Estas cifras reflejan niveles más altos de pobreza que la población puertorriqueña, donde las familias que viven en el nivel de pobreza alcanzan $45.4 \%$. La misma encuesta para el censo de Puerto Rico indica que $59.5 \%$ de la población tiene un ingreso anual menor a 24,999 dólares.

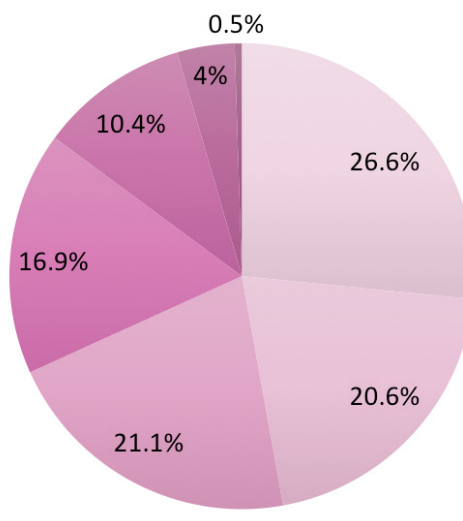

$$
\begin{aligned}
& \square \text { Menos de 5,000 dólares } \\
& \square \text { Entre 5,001 y 10,000 dólares } \\
& \square \text { Entre } 10,001 \text { y 20,000 dólares } \\
& \square \text { Entre } 20,001 \text { y 30,000 dólares } \\
& \square \text { Entre 30,001 y 50,000 dólares } \\
& \text { — Entre 50,001 y 100,000 dólares } \\
& \text { — Más de 100,000 dólares }
\end{aligned}
$$

\section{Figura 2.}

Participantes por renglón de ingresos anuales aproximados en el hogar (porcentaje).

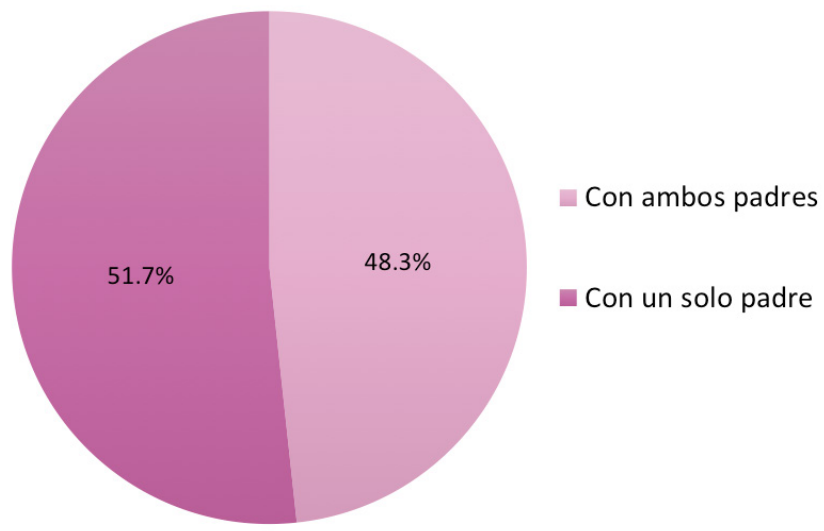

Figura 3.

Participantes que viven con ambos padres o con un solo padre (madre) (porcentaje).

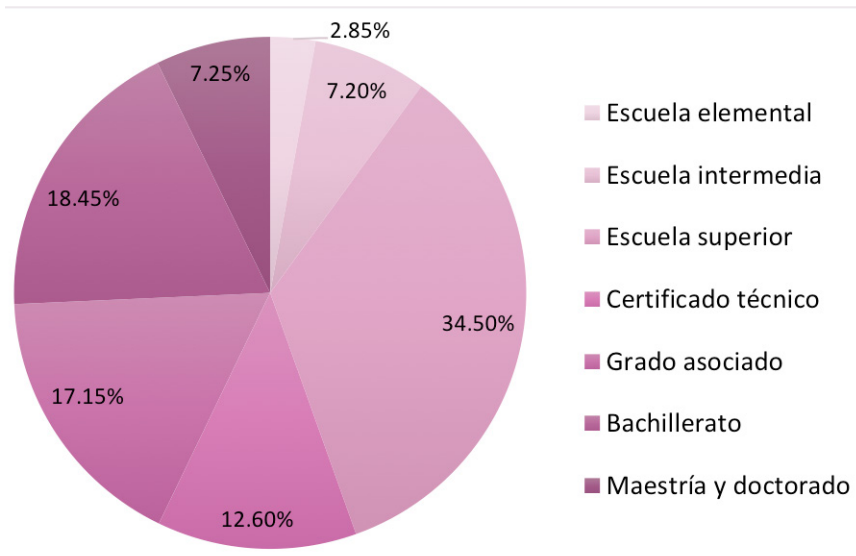

Figura 4.

Mayor grado académico alcanzado por los padres (madres).

\section{Materiales}

A los participantes se les administró el Cuestionario de Factores de Violencia, cuya la autoría es de la investigadora. Es un cuestionario de autoinforme donde los participantes escribían sus respuestas en el formulario provisto. Investigaciones similares (Posada y Parales, 2012) han demostrado que cuando la comunidad en que se insertan los examinados muestra alta violencia, los indicadores de nivel comunitario son consistentes entre los individuos; sin embargo, las experiencias de los jóvenes pueden variar de manera considerable en la frecuencia y gravedad de la exposición a dicha violencia. En esta situación, el autoinforme de exposición a la violencia es una manera indicada de evaluar la ecología del entorno (McMahon et al., 2013), ya que los indicadores del vecindario no arrojarán luz acerca de las diferentes formas en que los jóvenes han experimentado los niveles de exposición a la violencia de la comunidad. Además, los comportamientos de los estudiantes pueden ser diferentes en respuesta 
a un mismo entorno, y es es posible esperar actuaciones distintas de un mismo individuo en distintos niveles del sistema ecológico (Posada y Parales, 2012).

\section{Procedimiento}

Los participantes contestaron el Cuestionario de Factores de Violencia en sus escuelas. Previamente, el cuestionario fue validado por jueces expertos $(C V R=0.99) y$ pasó por un proceso de prueba piloto. Cada juez evaluó los reactivos del cuestionario en términos de claridad de su significado, su pertinencia en cuanto a la dimensión que pretendía medir, y tuvo la oportunidad de sugerir correcciones directas a los reactivos. Se hizo una ronda de administración para la prueba piloto con 45 estudiantes de una de las escuelas participantes. La prueba piloto incluía solicitar a los participantes que reportaran cualquier reactivo, contenido de las preguntas, instrucciones o formato de respuesta que les causara confusión o que fuera difícil de comprender. El contenido de las preguntas, así como las instrucciones y el formato de respuestas, resultaron ser comprensibles para los grupos de edad en la prueba piloto. El instrumento final contiene un formato de medición de preguntas cerradas (preguntas que hacen que el participante seleccione su respuesta entre un conjunto provisto de opciones) y escalas Likert de acuerdo, cantidad y frecuencia.

\section{Mediciones}

El cuestionario midió las dimensiones de violencia en la comunidad, violencia intrafamiliar, influencias negativas de los pares, inestabilidad emocional y conducta violenta.

\section{Percepción de violencia en la comunidad}

La escala de violencia en la comunidad está construida estilo Likert en una escala de cuatro puntos de frecuencia, con 10 reactivos en aseveraciones positivas. La evidencia de validez ha demostrado que el autoinforme de exposición a la violencia proporciona una indicación precisa de la cantidad de violencia a la que están expuestos los jóvenes (Voith, Gromoske y Holmes, 2014). Algunos ejemplos de variables utilizadas en la escala fueron "Te pueden asaltar" y "Ocurren crímenes", entre otros. Las opciones para responder a los reactivos van desde "Siempre" hasta "Nunca". Las variables fueron codificadas como Siempre $=3$, Muchas veces $=2$, Pocas veces $=1$ y Nunca $=0$. Al igual que en la escala anterior, las puntuaciones altas reflejan mayores niveles de violencia en la comunidad. Esta escala arrojó un índice de consistencia interna de $\alpha=0.913$.

\section{Ausencia de violencia intrafamiliar}

La escala de ausencia de violencia intrafamiliar consistió de 10 reactivos dispuestos en un arreglo Likert de frecuencia. Las opciones para responder a los reactivos fluctuaron desde "Siempre" hasta "Nunca". Las variables fueron codificadas como Siempre $=3$, Muchas veces $=2$, Pocas veces $=1$ y Nunca $=0$. Las puntuaciones altas reflejaban mayores niveles de violencia intrafamiliar y las puntuaciones más bajas denotaban menores niveles de esta variable. Las instrucciones dirigían a los participantes a responder si alguna de las personas con quien vive ha efectuado diferentes acciones, como "Te grita" y "Te pega en el cuerpo", entre otras. El índice de consistencia interna de esta escala fue $\alpha=0.815$.

\section{Influencias negativas de los pares}

La influencia negativa de los amigos se midió con una escala Likert de frecuencia de cuatro puntos. La escala consistió de cuatro reactivos, con variables, como "Mis amigos son personas violentas" o "Mis amigos tienen problemas con la ley". Los participantes podían seleccionar el nivel de frecuencia desde "Siempre" hasta "Nunca", con las puntuaciones altas denotando mayor influencia negativa de los pares y las puntuaciones bajas implicando menor influencia negativa de los amigos. Las variables fueron codificadas como Siempre $=3$, Muchas veces $=2$, Pocas veces $=1$ y Nunca $=0$. Esta medida de influencia negativa de amigos obtuvo un índice de consistencia interna de $\alpha=0.732$.

\section{Inestabilidad emocional}

Este aspecto incluyó indicadores de impulsividad, frustración, ansiedad, falta de control, facilidad para las alteraciones del ánimo y otros. Es una escala de 10 reactivos, igualmente dispuesta en arreglo Likert de cuatro puntos de frecuencia, definidos desde "Siempre" hasta "Nunca". Las variables fueron codificadas como Siempre $=3$, Muchas veces $=2$, Pocas veces $=1$ y Nunca $=$ 0 . Los reactivos incluyeron premisas como "Me pongo ansioso con facilidad" y "Me frustro con facilidad cuando algo me sale mal". El índice de consistencia interna de esta escala fue $\alpha=0.875$.

\section{Conducta violenta.}

La variable dependiente del estudio fue conducta violenta. Ésta se midió con una escala compuesta de indicadores de conducta agresiva (como "Incito a otros estudiantes a pelear", "Golpeo objetos cuando tengo coraje", "Insulto a las personas cuando me enojo con ellas") e indicadores de conducta antisocial (como "Destruyo a propósito cosas de otras personas", "Robo objetos", "Prefiero ignorar 
las normas establecidas"). Continuando el patrón de reactivos, esta escala también está dispuesta en un arreglo Likert de cuatro puntos de frecuencia, desde "Siempre" hasta "Nunca", codificadas como Siempre $=3$, Muchas veces $=2$, Pocas veces $=1$ y Nunca $=0$, donde las puntaciones altas denotan mayor grado de conducta violenta y las puntuaciones más bajas implican menor grado de conducta violenta. La escala consta de 20 ítems, con una consistencia interna de $\alpha=0.835$.

\section{AnÁlisis estadísticos}

El análisis de datos se efectuó utilizando modelos de ecuaciones estructurales (SEM, por sus siglas en inglés). Se calcularon estadísticas correlacionales bivariadas, medias y desviaciones estándar para las variables continuas y frecuencias para las variables del estudio. Además, como se ha mencionado, se computaron alfas de Cronbach para determinar la confiabilidad de las escalas. Después se especificó un modelo de ecuaciones estructurales en el que se estimaron los efectos directos en la conducta violenta de los jóvenes (variable endógena) para tres variables exógenas, "percepción de violencia en la comunidad" (exosistema), "ausencia de violencia intrafamiliar" (microsistema) e "influencia negativa de los pares" (microsistema). En este arreglo estructural de variables el mesosistema quedó representado por el supuesto de que las variables de microsistemas covarían, esto es, las covarianzas entre influencia negativa de los amigos y ausencia de violencia familiar entre ausencia de violencia familiar y percepción de violencia en la familia, y entre influencia negativa de los amigos y percepción de violencia en la familia. Se calcularon además los efectos indirectos de estas tres variables exógenas en la conducta violenta, siendo mediadas por la variable endógena "inestabilidad emocional".

Los modelos basados en regresiones lineales, como el presentado en este estudio, asumen el cumplimiento de los supuestos básicos para sus análisis estadísticos. En este caso, los datos reflejaron cumplir con dichos supuestos, con excepción en lo referente a la normalidad multivariada. Este es un problema típico al diseñar modelos estructurales cuyos datos son ordinales en su origen. La corrección de Satorra y Bentler ofrece una solución a la ausencia de normalidad multivariada que en la práctica corrige la situación para proceder sin dificultad al análisis estadístico. Todas las medidas y estimaciones reportadas en este estudio utilizan la corrección de Satorra y Bentler (1994, 2001, 2010).

\section{Resultados}

Para lograr los objetivos propuestos se han utilizado los paquetes estadísticos IBM SPSS Statistics 23, STATA v.13 y AMOS v.24. En primer lugar se han hecho análisis descriptivos (media, desviación típica), se ha obtenido la consistencia interna para las escalas utilizadas y se han calculado las correlaciones entre ellas. En la tabla 1 se muestran los totales de la muestra, valores máximos y mínimos, las medias y las desviaciones típicas, así como la consistencia interna de las escalas.

\section{Tabla 1.}

Total de la muestra, valores máximos, mínimos, medias, desviaciones estándar e índice de consistencia interna para las variables y escalas del estudio.

\begin{tabular}{|l|c|c|c|c|c|c|}
\hline \multicolumn{7}{|c|}{ N } \\
\hline $\begin{array}{l}\text { Influencias } \\
\text { negativas de } \\
\text { los pares }\end{array}$ & 431 & 0 & 15 & 1.64 & 2.203 & 0.732 \\
\hline $\begin{array}{l}\text { Ausencia } \\
\text { de violencia } \\
\text { intrafamiliar }\end{array}$ & 431 & 11 & 40 & 35.68 & 3.995 & 0.815 \\
\hline $\begin{array}{l}\text { Percepción } \\
\text { de violencia } \\
\text { en la } \\
\text { comunidad }\end{array}$ & 431 & 0 & 29 & 8.81 & 7.869 & 0.913 \\
\hline $\begin{array}{l}\text { Inestabilidad } \\
\text { emocional }\end{array}$ & 431 & 0 & 30 & 12.33 & 6.614 & 0.875 \\
\hline $\begin{array}{l}\text { Conducta } \\
\text { violenta }\end{array}$ & 431 & 0 & 42 & 7.99 & 6.275 & 0.835 \\
\hline $\begin{array}{l}\text { N válida } \\
\text { (listwise) }\end{array}$ & 431 & & & & & \\
\hline
\end{tabular}

Según se reportó, los índices de consistencia interna fluctuaron desde moderados altos hasta altos. Los análisis de correlación se muestran en la tabla 2. Se reportaron correlaciones de Pearson significativas para todas las variables del estudio con conducta violenta: influencias negativas de pares $(r=0.604, p<0.01)$, ausencia de violencia intrafamiliar $(r=$ p0.422, p < 0.01), percepción de violencia en la comunidad $(r=0.283, p<0.01)$, e inestabilidad emocional $(r=0.588, p$ $<0.01$ ). La influencia negativa de los pares y la inestabilidad emocional denotan índices marcadamente altos, sugiriendo una relación importante con las conductas violentas. Según se espera, la ausencia de violencia en la familia correlaciona de modo negativo con la conducta violenta de los jóvenes, arrojando un índice de asociación considerable. Llama la atención que todas las variables correlacionan significativamente entre sí al nivel de $p<0.01$, en especial la relación negativa que muestra la ausencia de violencia en la familia con la inestabilidad emocional, con un valor de $r=p 0.483$. 
Tabla 2.

Matriz de correlaciones para las variables influencias negativas de pares, ausencia de violencia intrafamiliar, percepción de violencia en la comunidad, inestabilidad emocional y conducta violenta.

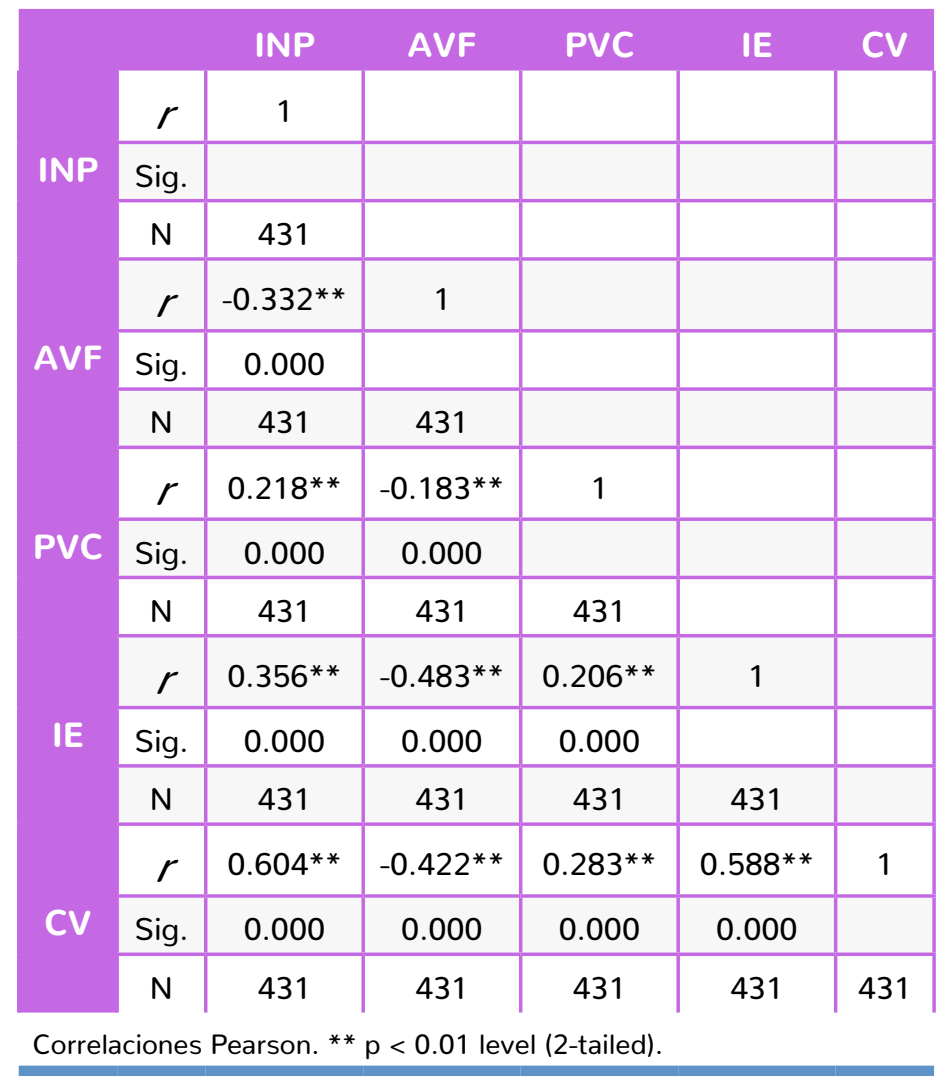

INP (influencia negativa de pares), AVF (ausencia de violencia intrafamiliar), PVC (percepción de violencia en la comunidad), IE (inestabilidad emocional), CV (conducta violenta).

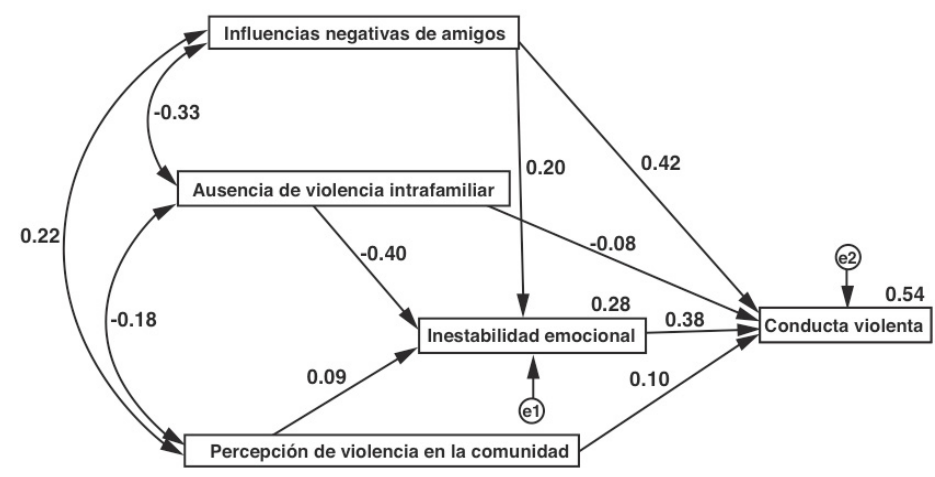

Figura 5.

Rutas del modelo propuesto de conducta violenta en jóvenes con índices para los parámetros estimados.

El sistema de relaciones que se nuestra en la figura 1 se evaluó con un modelo de ecuaciones estructurales, basado en covariancias y tratando a las variables como observables (análisis de rutas), utilizando el método de máxima verosimilitud. El modelo propuesto es uno jus- tamente identificado (Kline, 2011), por lo que los grados de libertad son igual a cero $(\mathrm{gl}=0)$ y Chi cuadrado es igual a $0(\mathrm{CMIN}=0)$. Se estimaron la magnitud y la dirección de los efectos directos, indirectos y totales de cada factor propuesto para el modelo utilizando los programas AMOS v.24 y STATA v.14. En la figura 5 se muestran las rutas para el modelo de factores propuesto, junto a los índices para los parámetros estudiados.

Todos los parámetros estimados del estudio resultaron significativos a $\mathrm{p}<0.01$, con excepción de la influencia de percepción de violencia en la sociedad sobre inestabilidad emocional y de ausencia de violencia en la familia sobre conducta violenta, las cuales resultaron significativas a $p$ $<0.05$. La varianza explicada para la variable endógena conducta violenta fue de $\mathrm{R}^{2}=0.54$, y para la variable endógena inestabilidad emocional fue de $R^{2}=0.28$, lo que muestra la alta influencia de las variables examinadas en la conducta violenta en jóvenes. Las pruebas de los parámetros individuales realizadas no mejoraron el modelo, y los índices de modificación fueron cero (0); por tanto, no hubo evidencia de que un modelo alternativo fuera mejor que un modelo justamente identificado.

Se puede observar que las influencias negativas de amigos y la percepción de violencia en la comunidad predijeron significativa y positivamente la inestabilidad emocional, con mayor peso de las influencias negativas de amigos $(0.20)(p<0.01)$ frente a la percepción de violencia en la comunidad (0.09) $(p<0.05)$. La ausencia de violencia intrafamiliar predijo significativamente $(p<$ 0.01 ), y de forma negativa e importante la inestabilidad emocional $(p$ 0.40). El total de varianza de la inestabilidad emocional explicada por la influencia negativa de los amigos, la ausencia de violencia intrafamiliar y la percepción de violencia en la comunidad fue de $28 \%$.

La influencia negativa de los pares tuvo un efecto directo significativamente positivo $(p<0.01)$ en la conducta violenta (0.42). Además, reflejó un efecto mediado por inestabilidad emocional en la conducta violenta (0.077), mostrando un importante efecto total (0.499) en esta variable endógena conducta violenta. Asimismo, la ausencia de violencia intrafamiliar ( $p 0.08)$ se mostró como predictor significativo $(p<0.05)$ y reductor de la conducta violenta. La ausencia de violencia en la familia tuvo a su vez un efecto indirecto mediante inestabilidad emocional en la conducta violenta ( $p$ 0.151) y demostró un efecto total amortiguador de la conducta violenta ( $p 0.232$ ).

La percepción de violencia en la comunidad, similar a la influencia negativa de los amigos, tuvo un efecto directo positivo $(0.10)(p<.01)$ en la conducta violenta en jóvenes. Además, la percepción de violencia en la comunidad se mostró como predictor de la conducta violenta 
de los jóvenes mediante inestabilidad emocional (0.034). El efecto total de la percepción de violencia en la comunidad (0.132) es positivo, indicando que en nuestra muestra aumentan las manifestaciones de conducta de violencia en jóvenes. Por su parte, la variable endógena inestabilidad emocional mostró un importante peso positivo en la conducta violenta de los jóvenes $(0.38)(p<0.01)$. En la tabla 3 se muestran los coeficientes estandarizados de los efectos directos, indirectos y totales de las variables del estudio en la conducta violenta.

\section{Tabla 3.}

Coeficientes estandarizados de los efectos directos, indirectos y totales para influencias negativas de pares, ausencia de violencia intrafamiliar, percepción de violencia en la comunidad, inestabilidad emocional en conducta violenta.

\begin{tabular}{|l|c|c|c|}
\hline \multicolumn{1}{|c|}{$\begin{array}{l}\text { EFECTOS } \\
\text { DIRECTOS }\end{array}$} & \multicolumn{1}{c|}{$\begin{array}{l}\text { EFECTOS } \\
\text { INDIRECTOS }\end{array}$} & $\begin{array}{c}\text { EFECTOS } \\
\text { TOTALES }\end{array}$ \\
\hline $\begin{array}{l}\text { Influencia negativa } \\
\text { de pares }\end{array}$ & 0.421 & 0.077 & 0.499 \\
\hline $\begin{array}{l}\text { Percepción de } \\
\text { violencia en la } \\
\text { comunidad }\end{array}$ & 0.099 & 0.034 & 0.132 \\
\hline $\begin{array}{l}\text { Ausencia de } \\
\text { violencia en la } \\
\text { familia }\end{array}$ & -0.081 & -0.151 & -0.232 \\
\hline $\begin{array}{l}\text { Inestabilidad } \\
\text { emocional }\end{array}$ & 0.378 & - & 0.378 \\
\hline
\end{tabular}

El modelo propuesto resultó cónsono con la base teórica implicada, indicando que la influencia negativa de los pares, la ausencia de violencia en la familia, la percepción que tenga el joven acerca de la violencia en su comunidad y su inestabilidad emocional son predictores importantes de la conducta violenta en jóvenes, logrando explicar 54\% de su varianza.

\section{Discusión}

El estudio demostró que —-desde distintos niveles ecológicos de influencia- los factores "violencia en la comunidad", "violencia intrafamiliar", "influencias negativas de los pares" e "inestabilidad emocional" son predictores importantes de las manifestaciones de la conducta violenta en jóvenes. Los datos se ajustan de manera favorable a los supuestos teóricos del modelo sistémico ecológico de Bronfenbrenner, sugiriendo niveles de influencia mayores según se hacen más próximos al individuo. Según la base teórica de la cual partimos, el poder de la influencia de un factor de orden superior será más débil que el de sistemas más cercanos al individuo y estará modulado por el estado emocional de la persona. En este caso en que hablamos de inestabilidad emocional, por la dirección negativa de este efecto para el individuo, la teoría sugiere que tendrá un efecto amplificador de la conducta violenta o reductor de los efectos protectores de la ausencia de violencia en la familia, y así se comprobó en este estudio.

Se destaca el efecto significativo que demuestran las influencias negativas de los pares en la conducta violenta de los jóvenes. La literatura ha evidenciado una mayor vulnerabilidad durante el proceso adolescente para iniciar conductas de riesgo en salud mental y adaptación social (Alarcón, Vinet y Salvo, 2005), incluyendo la influencia que puedan tener los amigos y los compañeros de escuela o vencidario en esta etapa del desarrollo. La influencia negativa de los amigos resultó más fuerte aún en presencia de inestabilidad emocional en la persona. Estos resultados son consecuentes con lo establecido por Bronfenbrenner cuando explica que los elementos microsistémicos se asocian con las características particulares del individuo, y éstas a su vez magnifican o atenúan el efecto de estos factores.

Era de esperar que el efecto de la ausencia de violencia en la familia en la conducta violenta de los jóvenes fuera negativo, esto es, que la ausencia de violencia en la familia debía funcionar como atenuante de la conducta violenta, tal como se reflejó en el estudio. Sin embargo, el mayor efecto de este factor fue en la inestabilidad emocional de los jóvenes, evidenciando un componente amortiguador de la misma. Otras investigaciones respaldan este hallazgo de la violencia familiar como predictora del estado emocional de la persona. Se ha observado un efecto directo en la depresión, en la ansiedad y en la conducta antisocial a partir de la exposición a violencia familiar (Frías y Gaxiola, 2008). La violencia familiar produce problemas psicosociales en los menores, manifestándose dichos problemas como de conducta, alteraciones psicológicas y problemas escolares. (Frías y Gaxiola, 2008). Estudios con variables relacionadas han evidenciado que los adolescentes que experimentan violencia filioparental muestran una mayor agresividad global, física e ira (Zuñeda, Llamazares, Marañón y Vázquez, 2016).

A su vez, se demostró que la inestabilidad emocional tiene consecuencias importantes en la conducta violenta de los jóvenes. Los adolescentes con problemas de impulsividad e inestabilidad emocional pueden ser menos propensos a guardar las precauciones necesarias para evitar conflictos y pueden presentar tendencia a no considerar las consecuencias de su comportamiento. 
Estudios que han analizado los efectos de la inestabilidad emocional (McMahon et al., 2013; Stoddard et al., 2012b) indican que para quienes muestran alta impulsividad se observa una relación inversa entre la exposición a violencia en la comunidad y el comportamiento prosocial, mientras que para quienes presentan baja impulsividad no se evidencia una relación entre la exposición a la violencia comunitaria y el comportamiento prosocial del joven. Por tanto, la inestabilidad emocional (que conlleva menor autocontrol por parte del joven) funciona como un factor de riesgo mediante la acentuación de la asociación positiva entre la exposición a la violencia comunitaria y la conducta violenta.

Por último, este estudio teorizó que el poder de los niveles superiores del entorno sería menor según se aleja del individuo en el sistema ecológico. Este supuesto se confirmó para la percepción de violencia en la comunidad, de nivel más externo que los demás factores de esta investigación, y con efecto menor reflejado, pero igualmente significativo. Los problemas del vecindario y la comunidad han sido destacados en otras investigaciones como factores de importancia en la predicción de la conducta agresiva y violenta, antes y después del ajuste para factores de riesgo a nivel individual, como uso y consumo de alcohol entre pares, falta de supervisión de adultos, depresión y otros datos demográficos (Jennings, Maldonado-Molina, Reingle y Komro, 2011). Por otro lado, Boxer et al. (2013) también han encontrado un efecto similar en la violencia etnopolítica, de nivel macrosistémico, y de la violencia en la comunidad, de nivel exosistémico, en el comportamiento violento en niños, mediado por factores de niveles microsistémicos, como la familia. Esto es, los factores de niveles más externos, como la percepción de violencia en la comunidad, de nivel exosistémico, afectarán el desarrollo de conductas violentas en jóvenes, tanto de modo directo como indirecto. Sin embargo, es esencial incorporar en la investigación de su efecto los factores amplificadores o reductores que se encuentran en niveles más próximos al joven, como pueden ser una familia protectora, un grupo de pares que ejerzan influencias positivas y el estado emocional de la persona. Es parte de los próximos pasos de esta investigación explorar directamente, junto con los actores de la comunidad estudiada, la relevancia social de los factores que aquí mostraron alta significancia estadística.

Para ampliar este estudio se recomienda la exploración detallada de las covariaciones de mesosistemas, esto es, la relación entre la influencia negativa de los amigos, la ausencia de violencia en la familia y la percepción de violencia en la comunidad, y cómo se afectan positiva o negativamente unas a otras. De la misma manera, se sugiere que se trabajen estos factores y factores adicionales utilizando arreglos estructurales distintos que permitan hacer análisis confirmatorios de los mismos, y la eventual verificación y selección de los modelos explicativos más acertados para cada contexto. Además, una recomendación que se desprende de la literatura revisada, y con la cual coincidimos, es la importancia de examinar no sólo las distintas formas en que se experimenta la violencia, sino también analizar evidencia de los modos en que los jóvenes razonan acerca de sus situaciones de conflicto interpersonal, pues es indudable que sus sistemas de representación orientan sus acciones individuales (Posada y Parales, 2012). Futuras investigaciones pueden se enfocar en los ciclos reproductores de violencia por medio de la asociación recíproca entre sistemas ecológicos, incluyendo indagar acerca de las circunstancias ontogénicas en que los jóvenes que viven en contextos violentos aceptan o aprueban el uso de medios violentos para resolver conflictos.

\section{CONCLUSIONES}

Bronfenbrenner explicaba que la investigación social tiene que ser reestructurante. Argüía que la investigación de la ecología del ser humano y su desarrollo debe incluir experimentos que impliquen la reestructuración innovadora de los sistemas ecológicos, que parten de ideologías y estructuras institucionales prevalecientes (Bronfenbrenner, 1977). Brofenbrenner planteaba la redefinición de objetivos, funciones y actividades de intervención a partir de las interconexiones entre sistemas anteriormente aislados entre sí por los estudios científicos. Las conclusiones de este trabajo apuntan hacia la dirección de factores esenciales que se deben considerar al momento de planificar intervenciones para la prevención de la violencia en jóvenes. En este estudio, la percepción del joven en cuanto a la violencia en su comunidad, la influencia que pueda recibir de sus pares, la violencia que presente su núcleo familiar y las consecuencias de estos factores en su estabilidad emocional explicaron $54 \%$ de la variabilidad en las manifestaciones de su conducta violenta. A su vez se demostró que la inestabilidad emocional de los jóvenes de la muestra podía ser explicada en $28 \%$ por la percepción que tenían de la violencia en su comunidad, la influencia de sus amigos y la violencia (o ausencia de violencia) que experimentaron en su familia. Los índices correlacionales significativos que mostraron entre sí todos los factores del estudio nos permiten considerar que éstos cambian y se transforman juntos, por lo que deben ser incluidos de modo íntegro en los trabajos comunitarios 
de prevención de violencia y en el fomento de culturas óptimas para el desarrollo de los individuos.

Los profesionales de la conducta nos debemos enfocar en estrategias integradoras que defiendan el derecho de los niños y los jóvenes a crecer en ambientes donde los otros significativos no representen peligro ni violencia. La familia (ya sea biológica o sustituta), los amigos y la comunidad aún son clave en el desarrollo de personas saludables que puedan alcanzar su máximo potencial más allá del riesgo que les representa la violencia en su entorno. El espacio comunitario se nos vuelve a presentar con sus múltiples posibilidades: como lugar de reproducción de la violencia que viven nuestros jóvenes, o como foro de encuentros solidarios que fomenten subjetividades colectivas que conduzcan a la paz y al desarrollo de todos. Urge considerar estos factores de la conducta violenta en jóvenes en las prácticas de intervención y en la planificación exitosa de proyectos y trabajos de prevención de los distintos profesionales de la conducta humana.

\section{Referencias}

Alarcón, P., Vinet, E., Salvo, S. (2005). Estilos de personalidad y desadaptación social durante la adolescencia. Psykhe, 14 (1), 3-16.

Borowsky, I. W., Widome, R., \& Resnick, M. D. (2008). Young people and violence. International Encyclopedia of Public Health, 6, 675-684.

Boxer, P., Huesmann, L. R., Dubow, E. F., Landau, S. F., Gvirsman, S. D., Shikaki, K., \& Ginges, J. (2013). Exposure to violence across the social ecosystem and the development of aggression: A test of ecological theory in the IsraeliPalestinian conflict. Child Development, 84 (1), 163-177. doi: 10.1111/j.1467-8624.2012.01848.x

Bronfenbrenner, U. (1994). Ecological models of human development. In International Encyclopedia of Education. Vol. 3, 2nd Ed. Oxford: Elsevier. Reprinted in: Gauvain, M. \& Cole, M. (eds.), Readings on the development of children, 2nd Ed. (1993, pp. 37-43). NY: Freeman.

Bronfenbrenner, U. (2002). La ecología del desarrollo humano. España: Paidós.

Bronfenbrenner, U. (1979). The Ecology of Human Development: Experiments by Nature and Design.Cambridge, MA: Harvard University Press.

Bronfenbrenner, U. (1977). Toward an experimental ecology of human development. American Psychologist, 32 (7), 513531. doi: 10.1037/0003-066X.32.7.513

Bronfenbrenner, U., \& Crouter, A. C. (1983). The evolution of environmental models in developmental research. In $\mathrm{P}$. H. Mussen (Series Ed.) \& W. Kessen (Vol. Ed.), Handbook of child psychology, Vol. 1: History, theory, methods (4th ed., pp. 357-414). New York: Wiley.

Burnette, M. L., Oshri, A., Lax, R., Richards, D., \& Ragbeer, S. N. (2012). Pathways from harsh parenting to adolescent antisocial behavior: A multidomain test of gender moderation. Development and Psychopathology, 24 (6), 857-870. doi: $10.1017 /$ S0954579412000417

Cedeño, L. A., Elias, M. J., Kelly, S., \& Chu, B. C. (2010). School violence, adjustment, and the influence of hope on lowincome, African American youth. American Journal of Orthopsychiatry, 80 (2), 213-226. doi: 10.1111/j.19390025.2010.01025.x

Cummings, E. M., Goeke-Morey, M. C., Merrilees, C. E., Taylor, L. K., \& Shirlow, P. (2014). A social-ecological, processoriented perspective on political violence and child development. Child Development Perspectives, 8(2), 8289. doi:10.1111/cdep.12067

De la Peña-Fernández, M. (2010). Conducta antisocial en adolescentes: Factores de riesgo y de protección (tesis doctoral). Universidad Complutense de Madrid, España.

DuRant, R. H., Cadenhead, C., Pendergrast, R. A., Slavens, G., \& Linder, C. W. (1994). Factors associated with the use of violence among urban Black adolescents. American Journal of Public Health, 84(4), 612-617. http://dx.doi. org/10.2105/AJPH.84.4.612

Edwards, B., \& Bromfield, L. M. (2009). Neighborhood influences on young children's conduct problems and pro-social behavior: Evidence from an Australian national sample. Children and Youth Services Review, 31 (3), 317-324. doi: 10.1016/j.childyouth.2008.08.005

Farrell, A. D., \& Sullivan, T. N. (2004). Impact of witnessing violence on growth curves for problem behaviors among early adolescents in urban and rural settings. Journal of Community Psychology, 32 (5), 505-525. doi: 10.1002/ jcop. 20016

Finkelhor, D., Ormrod, R. K., \& Turner, H. A. (2007). Polyvictimizationand trauma in a national longitudinal cohort. Development and Psychopathology, 19 (1), 149166. doi: $10.1017 /$ S0954579407070083

Frías Armenta, M., \& Gaxiola Romero, J. C. (2008). Consecuencias de la violencia experimentada directa e indirectamente en niños: Depresión, ansiedad, conducta antisocial y ejecución académica. Revista Mexicana de Psicología, 25 (2), 237-248.

Gorman-Smith, D., Henry, D. B., \& Tolan, P. H. (2004). Exposure to community violence and violence perpetration: The protective effects of family functioning. Journal of Clinical Child and Adolescent Psychology, 33 (3), 439-449. doi: 10.1207/s15374424jccp3303_2

Graber, J. A., Nichols, T., Lynne, S. D., Brooks-Gunn, J., \& Botvin, G. J. (2006). A longitudinal examination of family, friend, and media influences on competent versus problem behaviorsamong urban minority youth. Applied Developmental Science, 10 (2), 75-85. doi: 10.1207/ s1532480xads1002_3

Hernández Sampieri, R., Fernández Collado, C., \& Baptista Lucio, P. (2010). Metodología de la investigación (5a. ed.). México: McGraw-Hill.

Hong, J. S., \& Garbarino, J. (2012). Risk and protective factors for homophobic bullying in schools: An application of the social-ecological framework. Educational Psychology Review, 24 (2), 271-285.doi:10.1007/s10648-012-9194-y

Hong, J. S., Kral, M. J., Espelage, D. L., \& Allen-Meares, P. (2012). 
The social ecology of adolescent-initiated parent abuse: A review of the literature. Child Psychiatry \& Human Development, 43 (3), 431-454. doi: 10.1007/s10578-0110273-y

Instituto de Estadísticas de Puerto Rico (IEPR), Policía de Puerto Rico (PPR), División de Estadísticas de la Criminalidad (2015). Otras estadísticas sociales, demográficas y conexas. Recuperado de http://www.estadisticas. gobierno.pr/iepr/Estadisticas/InventariodeEstadisticas/ tabid/186/ctl/view_detail/mid/775/report_id/00975852e14a-4339-a6c8-ab23fd36d2a1/Default.aspx

Jennings, W. G., Maldonado-Molina, M. M., Reingle, J. M., \& Komro, K. A. (2011). A multi-level approach to investigating neighborhood effects on physical aggression among urban Chicago youth. American Journal of Criminal Justice, 36 (4), 392-407. doi:10.1007/s12103-011-9118-2

Keenan, K., Wroblewski, K., Hipwell, A., Loeber, R., \& StouthamerLoeber, M. (2010). Age of onset, symptom threshold, and expansion of the nosology of conduct disorder for girls. Journal of Abnormal Psychology, 119 (4), 689-698. doi: 10.1037/a0019346

Kline, R. B. (2011). Principles and practice of structural equation modeling. New York: Guilford Press.

Latman, R. D., \& Swisher, R. R. (2005). The interactive relationship among adolescent violence, street violence, and depression. Journal of Community Psychology, 33 (3), 355-371. doi: 10.1002/jcop.20051

Margolin, G., Vickerman, K. A., Oliver, P. H., \& Gordis, E. B. (2010). Violence exposure in multiple interpersonal domains: cumulative and differential effects. Journal of Adolescent Health, 47 (2), 198-205. doi: 10.1016/j. jadohealth.2010.01.020

McMahon, S. D., Todd, N. R., Martinez, A., Coker, C., Sheu, C. F., Washburn, J., \& Shah, S. (2013). Aggressive and prosocial behavior: community violence, cognitive, and behavioral predictors among urban African American youth. American Journal of Community Psychology, 51 (3), 407421. doi: 10.1007/s10464-012-9560-4

Posada-Gilé, R., \& Parales-Quenza, C. J. (2012). Violencia y desarrollo social: Más allá de una perspectiva de trauma. Universitas Psychologica, 11 (1), 255-267. Recuperado de http://www.redalyc.org/articulo.oa?id=64723234021

Resnicow, K., Ross-Gaddy, D., \& Vaughan, R. D. (1995). Structure problem and positive behaviors in African American Youths. Journal of Consulting and Clinical Psychology, 63 (4), 594-603.

Salzinger, S., Ng-Mak, D. S., Feldman, R. S., Kam, C., \& Rosario, M. (2006). Exposure to community violence: Processes that increase the risk for inner-city middle school children. Journal of Early Adolescence, 26 (2), 232-266. doi: 10.1177/0272431605285712

Satorra, A., \& Bentler, P. M. (1994). Corrections to test statistics and standard errors in covariance structure analysis. In A. von Eye \& C. C. Clogg (eds.), Latent Variables Analysis: Applications for Developmental Research. Thousand Oaks, CA: Sage Publications, Inc., pp. 399-419.
Satorra, A., \& Bentler, P. M. (2001). A scaled difference chisquare test statistics for moment structure analysis. Psychometrika, 66 (4), 507-514. doi:10.1007/BF02296192

Satorra, A., \& Bentler, P. M. (2010). Ensuring positiveness of the scaled chi-square test statistic. Psychometrika, 75 (2), 243248.doi: 10.1007/s11336-009-9135-y

Slattery, T. L., \& Meyers, S. A. (2014). Contextual predictors of adolescent antisocial behavior: The developmental influence of family, peer, and neighborhood factors. Child \& Adolescent Social Work Journal, 31 (1), 39-59. doi: 10.1007/s10560-013-0309-1

Stoddard, S. A., Zimmerman, M. A, \& Bauermeister, J. A. (2012). A longitudinal analysis of cumulative risks, cumulative promotive factors, and adolescent violent behavior. Journal of Research On Adolescence, 22 (3), 542-555. doi: 10.1111/j.1532-7795.2012.00786.x

Stoddard, S. A., Whiteside, L., Zimmerman, M. A., Cunningham, R. M., Chermack, S. T., \& Walton, M. A. (2013). The relationship between cumulative risk and promotive actors and violent behavior among urban adolescents. American Journal of Community Psychology, 51 (1), 57-65. doi: 10.1007/s10464-012-9541-7

Swick, K. J., \& Williams, R. D. (2006). An analysis of Bronfenbrenner's bio-ecological perspective for early childhood educators: Implications for working with families experiencing stress. Early Childhood Education Journal, 33 (5), 371-378. doi: 10.1007/s10643-006-0078-y

U.S. Department of Commerce (USDC). Economics and Statistics Administration. U.S. Census Bureau, Puerto Rico Community Survey (2012). Accedido el 28 de agosto de 2016, desde http://www.census.gov/programs-surveys/ acs/data/pums.html

Veltman, M. W. \& Browne, K. D. (2001). Three decades of child maltreatment research, implications for the school years. Trauma, Violence and Abuse, 2 (3), 215-239. doi: $10.1177 / 1524838001002003002$

Voith, L., Gromoske, A., \& Holmes, M. (2014). Effects of cumulative violence exposure on children's trauma and depression symptoms: A social ecological examination using fixed effects regression. Journal of Child \& Adolescent Trauma, 7 (4), 207-216. doi: 10.1007/s40653-014-0026-8

Von Bertalanffy, L. (1968). General System Theory: Foundations, Development, Applications. New York: George Braziller.

Zuñeda, A., Llamazares, A., Marañón, D., \& Vázquez, G. (2016). Características individuales y familiares de los adolescentes inmersos en violencia filio-parental: La agresividad física, la cohesión familiar y el conflicto interparental como variables explicativas. Revista de Psicopatología y Psicología Clínica, 21 (1), 21-33. doi: 10.5944/rppc.vol.21.num.1.2016.15021 


\section{Meta-Análisis del Artículo}




\section{Dimensión Cuantitativa}

\section{Perfil de Evaluación entre pares}
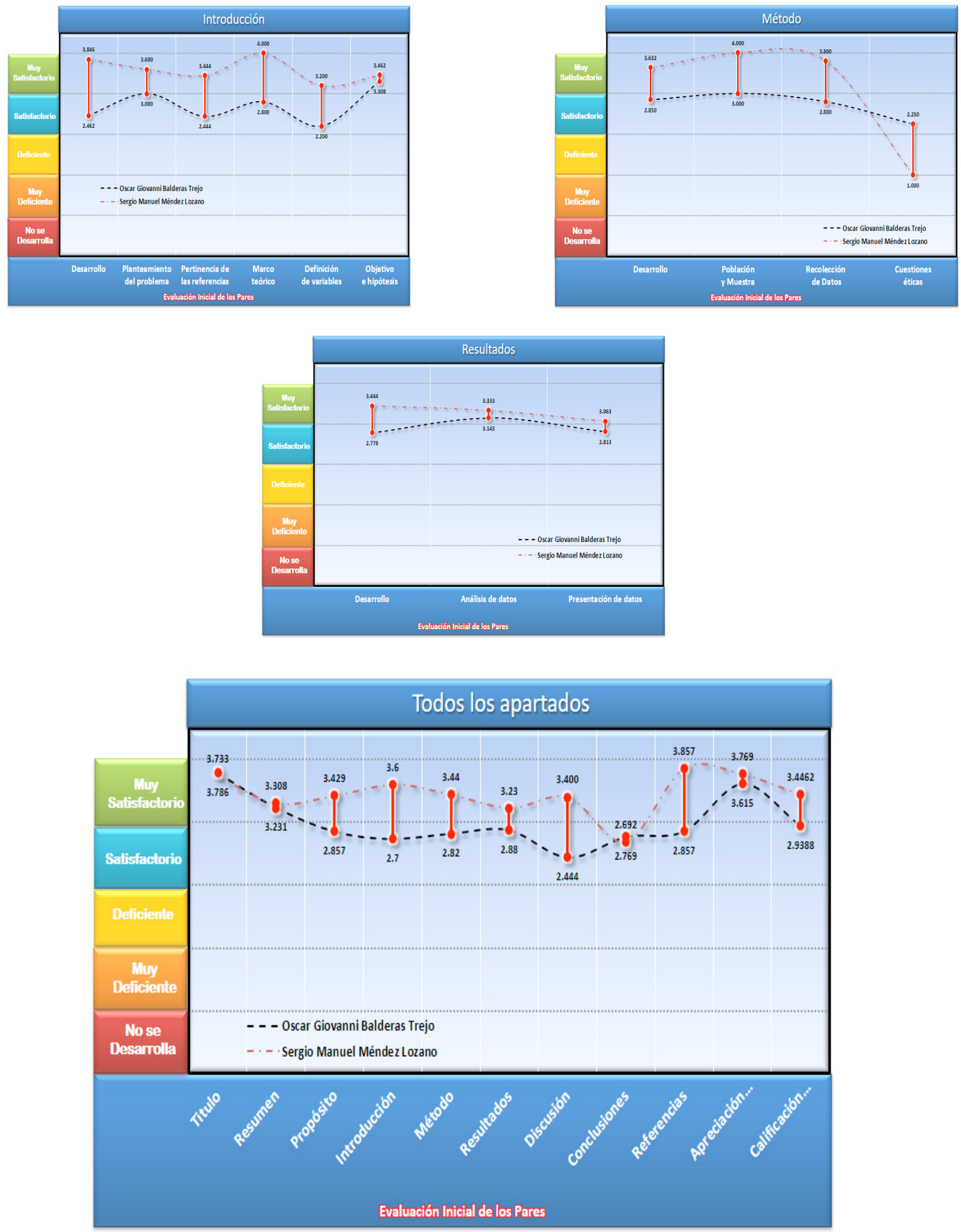


\section{Índice de Concordancia}

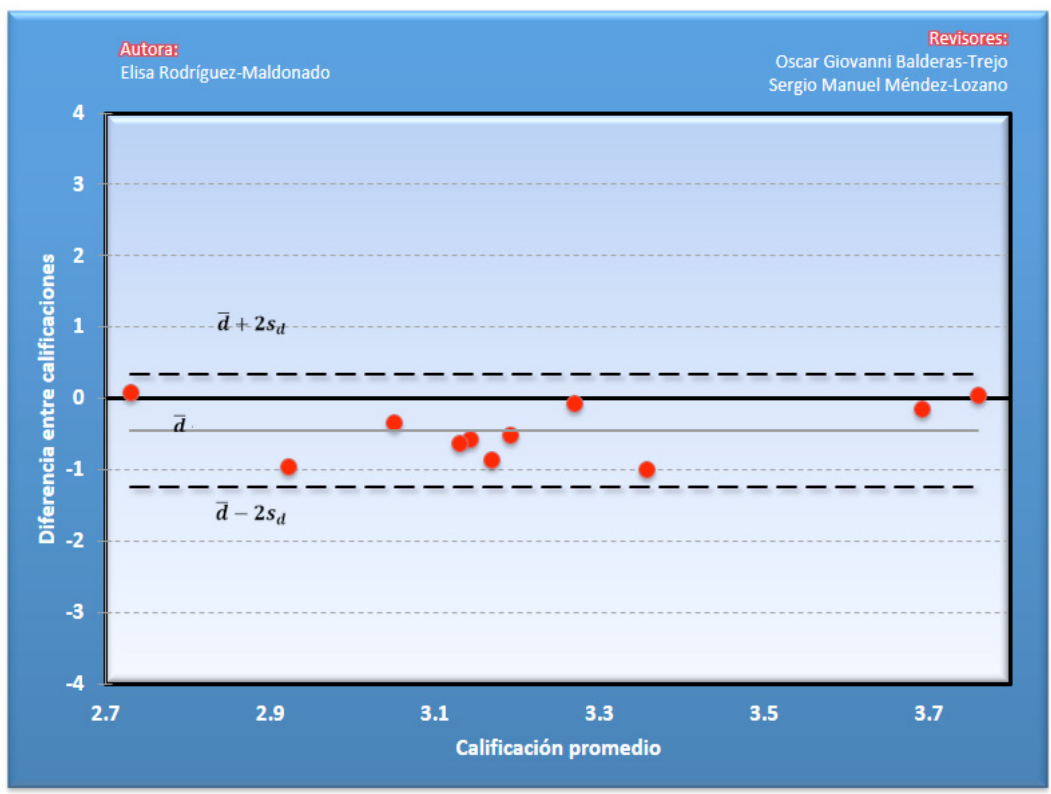

Índice de Acuerdo

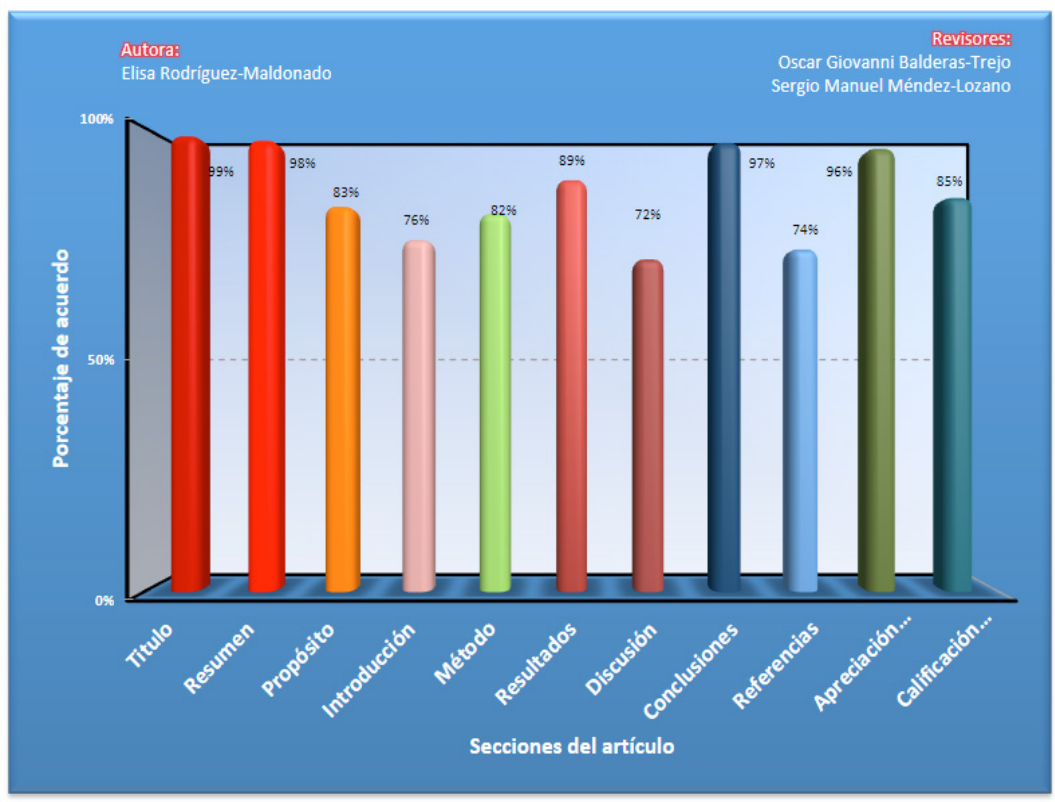


Dimensión Cualitativa

\begin{tabular}{|c|c|}
\hline Revisor 1 & Revisor 2 \\
\hline Sergio Manuel Méndez Lozano & Oscar Giovanni Balderas Trejo \\
\hline \multicolumn{2}{|c|}{ Título/Autoría } \\
\hline Ninguna & $\begin{array}{l}\text { El titulo se presenta de forma satisfactoria con relación a } \\
\text { los criterios establecidos por la APA y es congruente con } \\
\text { la investigación desarrollada }\end{array}$ \\
\hline \multicolumn{2}{|c|}{ Resumen } \\
\hline $\begin{array}{l}\text { Sugiero que se ajuste el resumen a } 150 \text { palabras y a } 5 \\
\text { palabras clave; y que se mencione que el SEM es a partir } \\
\text { de la aplicación de un cuestionario. Los resultados en } \\
\text { el resumen pueden ser más detallados con base en el } \\
\text { análisis realizado. }\end{array}$ & $\begin{array}{l}\text { La descripción de la metodología es planteada de forma } \\
\text { satisfactoria, sin embargo la discusión y conclusión del es- } \\
\text { tudio se muestra ambigua en el resumen. }\end{array}$ \\
\hline \multicolumn{2}{|c|}{ Próposito del Estudio } \\
\hline $\begin{array}{l}\text { Se sugiere que se puntualice la pregunta de investiga- } \\
\text { ción y que se mencione qué sucede con las variables } \\
\text { relacionadas con contextos similares a los de la muestra } \\
\text { seleccionada. }\end{array}$ & $\begin{array}{l}\text { El objetivo pareciera estar planteado de forma sesgada, } \\
\text { ya que se establece como hecho los resultados esperados } \\
\text { y el estudio unicamente funge un papel confirmatorio. }\end{array}$ \\
\hline \multicolumn{2}{|c|}{ Introducción } \\
\hline $\begin{array}{l}\text { Al inicio cuando se menciona a Bronfenbrenner se su- } \\
\text { giere diálogo con otros autores. Revisar redacción años } \\
\text { ' } 40 \text { y los siglos en número romano. Se invita a enfatizar } \\
\text { la relación que guardan en la teoría las relaciones de las } \\
\text { variables estudiadas con la violencia. }\end{array}$ & $\begin{array}{l}\text { Las referencias empleadas permiten la construcción de } \\
\text { conceptos claros, los cuales son resultan importantes en } \\
\text { el modelo de investigación empleado, sin embargo estos } \\
\text { no son desarrollados, el orden parece ser arbitraria ya no } \\
\text { se relaciona de forma directa una cita con la subsecuente } \\
\text { y hay referencia que podrían vincularse con el tema de in- } \\
\text { vestigación pero no se elabora satisfactoriamente. }\end{array}$ \\
\hline
\end{tabular}




\begin{tabular}{|c|c|}
\hline Revisor 1 & Revisor 2 \\
\hline \multicolumn{2}{|c|}{ Método } \\
\hline $\begin{array}{l}\text { Se sugiere mencionar las consideraciones éticas que se lle- } \\
\text { varon a cabo para realizar la investigación. Asimismo, se } \\
\text { invita a que del Cuestionario utilizado se mencionen a qué } \\
\text { refieren las preguntas cerradas y cuál es el tipo de validez } \\
\text { que presenta.Se invita a qué se mencione el tiempo que se } \\
\text { requirió para la recolección de datos y el procedimiento } \\
\text { llevado a cabo para el ingreso a las escuelas. }\end{array}$ & $\begin{array}{l}\text { Se utilizó un instrumento elaborado por la autora del ar- } \\
\text { ticulo, el cual no se presenta; se menciona que paso por } \\
\text { un proceso de validación, sin embargo no se describe con } \\
\text { precisión los resultados obtenidos de la validación. }\end{array}$ \\
\hline \multicolumn{2}{|c|}{ Resultados } \\
\hline $\begin{array}{l}\text { Se sugiere que se mencione con énfasis la magnitud de las } \\
\text { correlaciones encontradas, así como mayor especificidad } \\
\text { en la explicación de la varianza explicada. De igual forma, } \\
\text { se sugiere que se mencione puntualmente el valor de la chi } \\
\text { cuadrada. Es necesaria la mención de los índices de ajuste } \\
\text { absoluto y los índices de ajuste incremental, ya que no se } \\
\text { mencionan en el artículo, y no se logra , por lo tanto ob- } \\
\text { servar si es el modelo estructural propuesto el más óptimo. }\end{array}$ & $\begin{array}{l}\text { Se hace omisión de gráficos que podían facilitar la lectura } \\
\text { de los resultados obtenidos. }\end{array}$ \\
\hline \multicolumn{2}{|c|}{ Discusión } \\
\hline $\begin{array}{l}\text { Se sugiere que se argumenten los resultados puntuales } \\
\text { trabajados en la sección anterior, ya que a mi parecer se } \\
\text { abordan de manera bastante general. }\end{array}$ & $\begin{array}{l}\text { Como se menciono anteriormente, el trabajo cumple con } \\
\text { un papel confirmatorio de lo establecido previamente, por } \\
\text { lo que debería de presentarse como una discusión juega el } \\
\text { papel de una continuación del marco de referencia, donde } \\
\text { se confirma nuevamente los esperado, sin hacer contraste } \\
\text { de la información. }\end{array}$ \\
\hline \multicolumn{2}{|c|}{ Conclusiones } \\
\hline $\begin{array}{l}\text { Se sugiere mencionar las limitaciones que puede pre- } \\
\text { sentar el estudio, así como de la interpretación pun- } \\
\text { tual del modelo, junto con las variables involucradas. } \\
\text { Asimismo, se invita a realizar la distinción entre sig- } \\
\text { nificancia estadística y relevancia social; y enfatizar las } \\
\text { contribuciones del escrito y proponer reflexión sobre } \\
\text { investigaciones futuras. }\end{array}$ & $\begin{array}{l}\text { Se propone la aumentar la amplitud de lo analizado para } \\
\text { investigaciones futuras, sin embargo no se destaca el papel } \\
\text { de los resultados presentes en la investigación actual, si bien } \\
\text { se menciona que deberían de retomarse elementos para la } \\
\text { elaboración de intervenciones futuras sobre el tema, la con- } \\
\text { clusión no esclarece como participan los resultados obteni- } \\
\text { dos de forma novedosa en una futura intervención. }\end{array}$ \\
\hline \multicolumn{2}{|c|}{ Referencias } \\
\hline Revisar algunas citas con base en criterios APA. & $\begin{array}{l}\text { La mayoría de las referencias tienen alrededor de } 7 \text { años } \\
\text { de antigüedad, por lo que valdría la pena actualizar la in- } \\
\text { formación en algunos rubros. }\end{array}$ \\
\hline
\end{tabular}




\section{Historia del Proceso}

\section{EDITORIAL}

